\title{
Method of investigation of local corrosion processes on samples from clad steel
}

\author{
Dina Latypova ${ }^{1, *}$ and Oleg Latypov ${ }^{1}$ \\ ${ }^{1}$ Ufa State Petroleum Technological University, the Chair «Technology of Petroleum Devices», Ufa, Russia
}

\begin{abstract}
The article describes a method developed to evaluate the mechanism of local corrosion processes on the surface of a clad layer of steel specimens, which makes it possible to identify the difference in electrode potentials in artificially created holes. As a result of the study it was found that in a corrosive medium containing $\mathrm{Cl}^{-}$and $\mathrm{S}_{2} \mathrm{O}_{3}{ }^{2-}$ ions, the diffusion of oxidants into a hole of smaller diameter is difficult, which causes a large potential difference between the wall of the hole and its bottom. This process contributes to the development of local electrochemical corrosion.
\end{abstract}

\section{Introduction}

The quality of diesel fuel can be improved by applying hydrogenation processes. Hydrogenolysis of heteroorganic compounds in hydrofining processes occurs as a result of breaking the $\mathrm{C}-\mathrm{S}, \mathrm{C}-\mathrm{N}, \mathrm{C}-\mathrm{O}$ bonds and saturating the resulting heteroatoms with hydrogen and a double bond in the hydrocarbon portion of the crude oil molecules. At the same time, simple elements such as nitrogen, sulfur and oxygen are released as compounds, respectively $\mathrm{H}_{2} \mathrm{~S}, \mathrm{NH}_{3}, \mathrm{H}_{2} \mathrm{O}$. The impact of the most aggressive of them leads to the destruction of the passive layer of stainless steel, which leads to the development of local corrosion of oil refining and petrochemical equipment [1].

Currently, several methods are used to protection against corrosion of oil refining equipment: electrochemical protection, corrosion inhibitors, a clad layer of corrosion-resistant material [2]. In a diesel hydrotreatment unit, a cladding layer made of corrosionresistant steel is most often used to protect the upper body of the stripping column. This layer is subject to significant damage due to pitting corrosion. During the processing of high-sulfur oil, chlorine ions $\mathrm{Cl}^{-}$and thiosulfate ions $\mathrm{S}_{2} \mathrm{O}_{3}{ }^{2-}$ can stimulate the destruction of the metal, forming deep ulcers and pittings in some areas, which provoke further destruction of the apparatus due to the ulcer corrosion of the body made of carbon steel $[3,4]$. It was noticed that the pitting corrosion in aggressive media takes place in conjunction with the process of decarburization of the body of the apparatus made of carbon steel, which can lead to a loss of structural strength of the column. Analysis of corrosion factors showed that the main cause of decarburization is the residual water content in the hydrogenation product entering the column [5]. Therefore, the improvement of methods for protecting equipment from hydrotreating diesel fuel from pitting corrosion is an important condition for the high-quality and efficient processing of sour crude oil.

\section{Research and results}

A necessary condition for the occurrence of local corrosion is the presence of an oxidizing agent and an activator in a corrosive media, which contribute to the establishment of a certain value of the redox potential of the system, provoking the "breakdown" of a passive film of a corrosion-resistant layer of clad steel [6-7]. To evaluate the mechanism of the local corrosion process, samples of clad steel $09 \mathrm{Mn} 2 \mathrm{Si}+08 \mathrm{Cr} 13$ with holes of the same depth and different diameters were used, simulating the end-to-end destruction of the cladding layer due to the development of pitting or ulcers (Fig. 1).

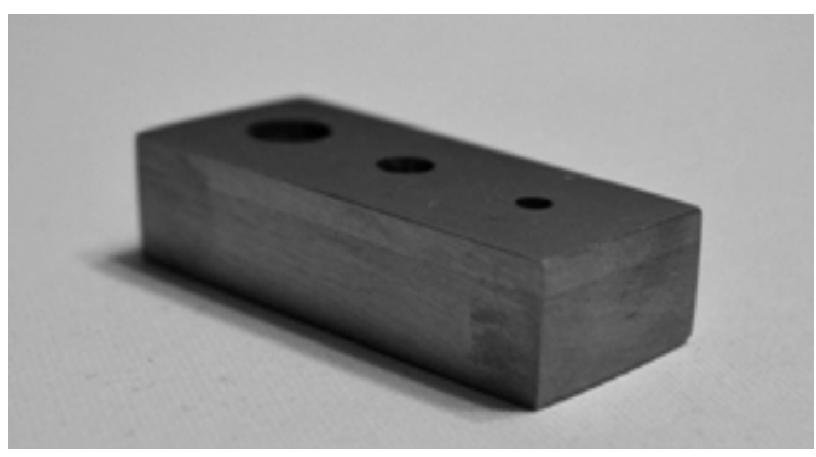

Fig. 1. Test specimen.

A solution was used as a corrosive medium $3 \%$ $\mathrm{NaCl}+3 \% \mathrm{H}_{2} \mathrm{SO}_{4}$. This solution was a model of the refining media, dominated by $\mathrm{Cl}^{-}$and $\mathrm{S}_{2} \mathrm{O}_{3}{ }^{2-}$ ions. Using a silver chloride reference electrode, we measured the electrode potentials of the surface of metal samples with a cladding layer near the holes and in accordance with Fig. 2. Fig. 3 shows the scheme for measuring the electrode potential on the surface of clad steel in a corrosive solution.

\footnotetext{
*orresponding author: d.r.latypova@mail.ru
} 




1-15 - Points for measurement

Fig. 2. Points for measurement electrode potential.

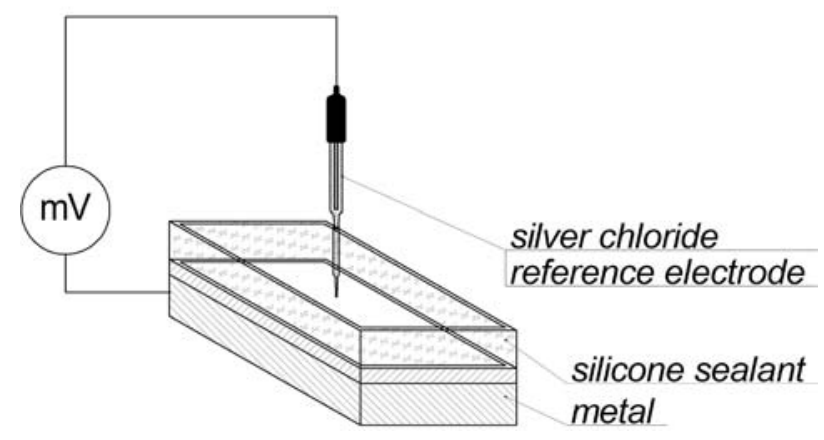

Fig. 3. The scheme for measuring the electrode potential on the surface of clad steel.

The results of measurements of electrode potentials are shown in Table 1.

Table 1. The results of measurements of electrode potentials $(\mathrm{mV})$ of the surface of a sample of steel $09 \mathrm{Mn} 2 \mathrm{Si}+08 \mathrm{Cr} 13$.

\begin{tabular}{|c|c|c|c|c|c|c|c|c|c|}
\hline \multicolumn{4}{|c|}{ Hole $\varnothing 7 \mathrm{~mm}$} & \multicolumn{5}{c|}{ Hole $\varnothing 5 \mathrm{~mm}$} \\
\hline 1 & 2 & 3 & 4 & 5 & 6 & 7 & 8 & 9 & 10 \\
\hline 0.451 & 0.415 & 0.391 & 0.413 & 0.450 & 0.449 & 0.422 & 0.418 & 0.421 & 0.450 \\
\hline
\end{tabular}

\begin{tabular}{|c|c|c|c|c|}
\hline \multicolumn{5}{|c|}{ Hole $\varnothing 3 \mathrm{~mm}$} \\
\hline 11 & 12 & 13 & 14 & 15 \\
\hline 0.447 & 0.376 & 0.325 & 0.378 & 0.448 \\
\hline
\end{tabular}

The experimental results showed a significant displacement of the electrode potential of the cladding layer in the area of the anodic dissolution of the metal (by $123 \mathrm{mV}$ ) when approaching a hole with a diameter of $3 \mathrm{~mm}$. The analysis of the obtained results made it possible to reveal the dependence of the amplitude of change of the electrode potentials of the clad steel on the hole diameter (Fig. 4).

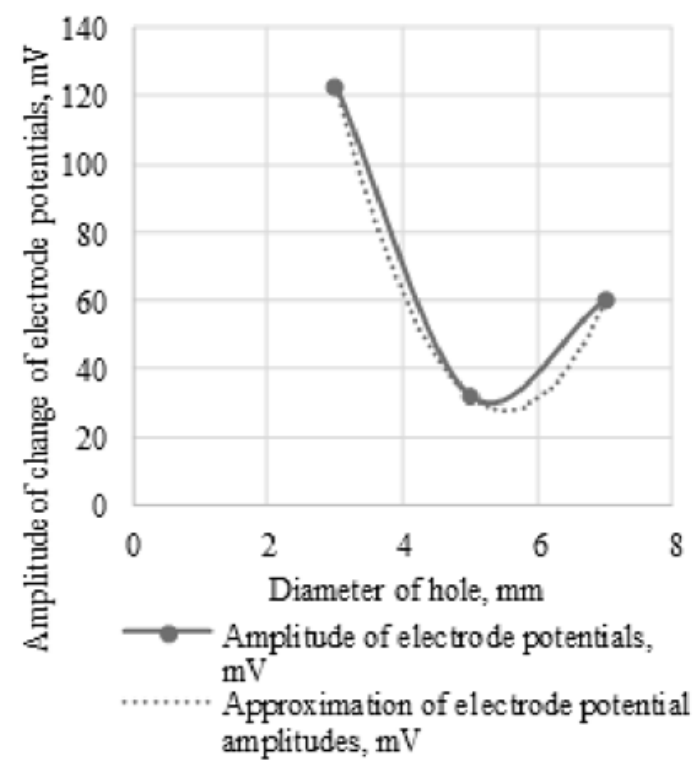

Fig. 4. Analysis of the dependence of the amplitude of the electrode potentials on the diameter of the hole.

Approximation of the dependence of the amplitude of the electrode potential difference on the hole diameter (Fig. 3) showed that with increasing hole diameter (up to $5.5 \mathrm{~mm}$ ), the difference in electrode potential between the metal surface and the bottom of the hole decreases, i.e. the likelihood of local corrosion is reduced. The dependence of the amplitude of the electrode potential difference on the hole diameter is parabolic. According to the above dependence, with the development of pitting with a hole diameter of up to $0.1 \mathrm{~mm}$, the potential difference between the surface of the cladding layer and the bottom can reach up to $466 \mathrm{mV}$, i.e. almost 4 times more than with the development of peptic ulcer corrosion, respectively, and the dissolution rate of the local galvanic area will be much higher. The decrease in the amplitude of the potential difference with an increase in the hole diameter (in the case of a hole with a diameter of $5 \mathrm{~mm}$ ) is caused by an increase in the concentration of corrosion products. If there are holes of larger diameter (hole diameter $7 \mathrm{~mm}$ ), the difference in electrode potentials increases again. Probably, under conditions of a large hole, better diffusion of oxidants to the surface of the $09 \mathrm{Mn} 2 \mathrm{Si}$ steel (inside the hole) is realized, which again enhances the development of local (contact) corrosion.

Thus, the destruction of the cladding layer of the column contributes to a significant increase in the potential difference between the surface of the layer and the body, made of carbon steel. The study of the mechanism of electrochemical processes on the surface of clad steel is necessary conditions for determining the conditions of polarization of a metal surface in order to reduce its corrosion rate [8-10]. Timely monitoring of the corrosion state of the cladding layer, under the conditions of the action of the pitting-forming ions, will prevent the destruction of the metal of the column. 


\section{Conclusions}

The study of the mechanism of local corrosion processes on the sample surface from clad steel $09 \mathrm{Mn} 2 \mathrm{Si}+08 \mathrm{Cr} 13$ made it possible to establish that diffusion of the oxidizer into the hole of a smaller diameter is difficult in an aggressive media containing $\mathrm{Cl}^{-}$and $\mathrm{S}_{2} \mathrm{O}_{3}{ }^{2-}$ ions, causing a large potential difference between the hole wall and its bottom.

Electrode potentials, measured near a hole of $3 \mathrm{~mm}$ in diameter, are displaced much more intensively (by $123 \mathrm{mV}$ ) than potentials measured near large holes of 5 and $7 \mathrm{~mm}$ in diameter. The electrode potential difference around the hole of diameter $7 \mathrm{~mm}$ is higher than that of the hole of $5 \mathrm{~mm}$. Under conditions of a large hole, better diffusion of oxidants to the surface of steel 09Mn2Si (inside the hole) is realized, which enhances the development of local (contact) corrosion. However, the large area of the contacting surface of the anode contributed to a decrease in the density of the corrosion current, which led to a lower corrosion rate than in a hole with a diameter of $3 \mathrm{~mm}$.

The developed method allows one to predict the corrosive behavior of the cladding layer of a column under the conditions of action of pitting-forming ions, and to prevent the destruction of the metal of the column.

\section{References}

1. D. Latypova, O. Latypov, D. Bugai, Nanotechnologies in Construction, Vol.10, no.3. 167-178 (2018)

2. A. Scherbakov, I. Kasatkina V., A. Palicheva, Corrosion: materials, protection, № 9, 31-36 (2017)

3. O. Latypov, D. Bugai, E. Boev, Chem. and Petrol. Eng. Vol.51, Iss.3, 283-285 (2015)

4. S. Cherepashkin, D. Latypova, E. Boev, Problems of collection, preparation and transportation of oil and petroleum products, № 4 (114), 73-84 (2018)

5. I. Kostitsyna, A. Shakhmatov, A. Davydov, E3S Web Conf., 121, 04006 (2019)

6. A. Altsibeeva V. Burlov, T. Kuzinova, Corrosion: materials, protection, № 3, 6-10 (2009)

7. I. Golubev, A. Laptev, E. Alekseeva, N. Shaposhnikov, A. Povyshev, M. Kurakin E3S Web Conf., 121, 02006 (2019)

8. O. Latypov, f Oilfield Equipment by Management of Electrochemical Parameters of the Medium Chem. and Petrol. Eng. Vol.51, Iss.7. 522-525 (2015)

9. N. Skuridin, D. Latypova, M. Pechenkina, O. Latypov, D. Bugai, V. Ryabukhina, Oil industry. 5, 84-86 (2018)

10. D. Latypova, Oil and gas business, T. 17, № 3. 6873 (2019) 